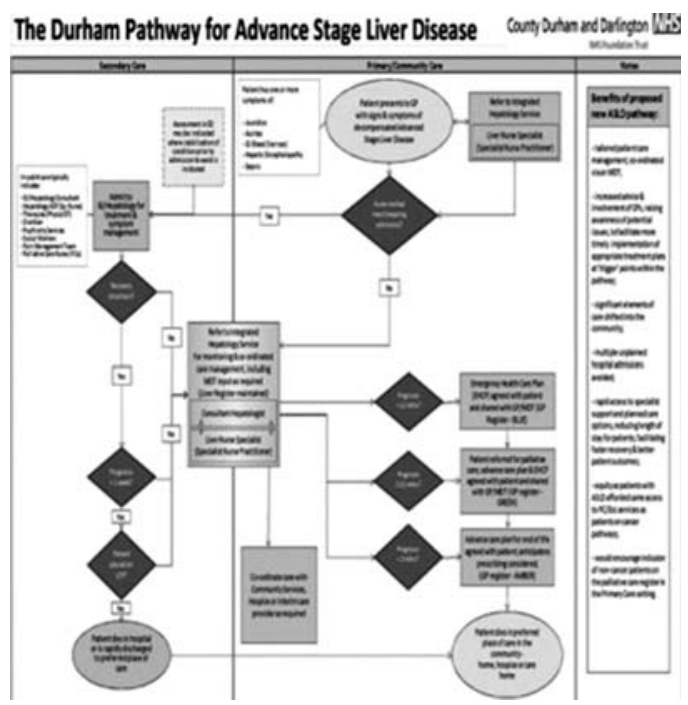

Abstract PTU-134 Figure 1

coordinated, and quality of life of patient and carer was poor. All post-pilot metrics reported significant improvements. Improved efficiency evidenced by reduction in unplanned hospital admissions, increase use of alternative community services, with use of shared care plans. $83 \%$ of these achieved their preferred place of care and death in contrast to nil pre pilot. Conclusion This pathway worked for ASLD and needs wider evaluation and consideration of similar approaches to other groups. Disclosure of Interest None Declared.

\section{PTU-135 ELAAR (THE ENHANCED LIVERPOOL ABDOMINAL AREA RATIO): HOW TO USE CROSS-SECTIONAL IMAGING TO ASSESS PROGNOSIS IN END-STAGE CIRRHOSIS}

${ }^{1}$ SM Alam*, ${ }^{2} \mathrm{C}$ Farrell, ${ }^{3}$ I Patanwala, ${ }^{3} \mathrm{P}$ Richardson, ${ }^{3} \mathrm{~T}$ Cross. ${ }^{1}$ Gastroenterology, Royal Liverpool and Broadgreen University Hospital Liverpool UK; ${ }^{2}$ Radiology, Royal Liverpool University Hospital, Liverpool, UK; ${ }^{3}$ Gastroenterology, Royal Liverpool University Hospital, Liverpool, UK

\subsection{6/gutjpl-2014-307263.209}

Introduction Liver size may be important in prognostication in cirrhosis. The LAAR score has previously shown a relationship between liver size and survival but is hampered by subjectivity. The current study aimed to improve the existing score.

Methods A retrospective-prospective cohort study was performed on patients with cirrhosis. The censor point used was date of patient death or liver transplant (LT) from CT date. Time points were measured from the date of the CT scan to censor point or last clinic appointment. The enhanced Liver ${ }^{\text {pool }}$ to Abdomen Area Ratio (eLAAR) was derived using a software package (Carestream). eLAAR was calculated using the formula (Liver area $\left(\mathrm{cm}^{2}\right)$ /Abdominal area $\left(\mathrm{cm}^{2}\right) \times 100$.

Results 101 patients were identified, 66\% male, median age 52 (44-60 years). The LAAR score detected progression to death/ LT in our cohort at 1 year $(\mathrm{p}=0.02)$ and at 5 years $(\mathrm{p}=0.03)$. The intra-class correlation coefficient between 2 operators was 0.94 (95\% CI 0.89-0.97). Using an optimal eLAAR cut-off of 32 eLAAR could predict death at 1 and 5 years from diagnosis, $\mathrm{p}=0.03($ OR 2.51(1.08-2.51) and $\mathrm{p}=0.002() \mathrm{R} 3.9895 \%$ CI 1.5-10.4). Survival curves were constructed and the log rank test showed that eLAAR was able to predict death at 1 year ( $\log$ rank 5.3, $\mathrm{p}=0.02)$ and 5 years (log-rank, $\mathrm{p}=9.7, \mathrm{p}=0.002)$.
Conclusion The eLAAR score offers a new paradigm to identify patients with poor prognostic criteria on cross-sectional imaging who may benefit from liver transplantation.

Disclosure of Interest None Declared.

\section{PTU-136 DOES A NORMAL IGG INDICATE HISTOLOGICAL REMISSION IN AUTOIMMUNE HEPATITIS (AIH)?}

'VM Gordon*, 'E McFarlane, ${ }^{2} \mathrm{~A}$ Dube, ${ }^{1} \mathrm{D}$ Gleeson. ${ }^{1}$ Hepatology, Sheffield Teaching Hospitals NHS Trust, Sheffield, UK; ${ }^{2}$ Histopathology, Sheffield Teaching Hospitals NHS Trust, Sheffield, UK

\subsection{6/gutjnl-2014-307263.210}

Introduction Response to immunosuppressive treatment in $\mathrm{AIH}$ is often monitored by measurement of serum immunoglobulin $G$ (IgG) as well as ALT. It is commonly assumed that serum IgG level correlates with histological activity (or Ishak necroinflammatory score: NIS) on liver biopsy, the historical "gold standard". However, only one group (Luth et al 2008; J of Clin 42 (8):926-930.) have examined this relationship, finding that normalisation of both serum ALT and IgG reliably predicted a NIS of $<6$ but not a NIS of $<4$ (corresponding to minimal hepatitis, seen in less than half of the patients presumed to be in remission). Here, we aimed to reassess how well serum IgG correlated with NIS in treated patients with AIH undergoing follow-up biopsy for confirmation of disease remission.

Methods We assessed 31 follow-up biopsies, performed to confirm histological remission in 28 patients with AIH (International Group criteria; Alvarez J Hepatol 1999; 31:929) on immunosuppressive treatment and an accompanying serum IgG (measured within 6 weeks of biopsy).

Results For 29 of the 31 follow-up biopsies, accompanying serum IgG was in the normal range ( $\leq 16 \mathrm{gm} / \mathrm{L})$. However, only 13 of these 31 biopsies showed NIS $<4$ (minimal hepatitis). On ROC analysis, area under the curve (AUC) for IgG in predicting a NIS of $\geq 4(\mathrm{n}=31)$ was $0.596(\mathrm{p}=0.368)$. Sensitivity and specificity of IgG (cut off $>16 \mathrm{grm} / \mathrm{L}$ ) in predicting a NIS of $\geq 4$ was $5.5 \%$ and $57 \%$ respectively. Corresponding positive (PPV) and negative (NPV) predictive values were only 50\% and 59\% respectively. AUC for change in $\operatorname{IgG}(\triangle \mathrm{IgG}$ : baseline values minus values accompanying follow up biopsy; $\mathrm{n}=29$ ) in predicting NIS $>4$ was $0.551(\mathrm{p}=0.642)$.

Defining histological remission instead as minimal or mild hepatitis- NIS < 6 (as Luth's group did because they found that such patients did not develop fibrosis progression), there were still 4/29 (14\%) patients with normal serum IgG who were not in histological remission. On ROC analysis, AUC for IgG in prediction of NIS $>6$ on follow-up biopsy $(\mathrm{n}=31)$ was $0.62(\mathrm{p}=$ 0.39 ). PPV and NPV for serum IgG (cut off $>16 \mathrm{grm} / \mathrm{L}$ ) in predicting of NIS $\geq 6$ ) was $50 \%$ and $86 \%$ respectively. AUC for $\Delta \mathrm{IgG}$ in prediction of NIS $\geq 6$ was 0.608 ( $\mathrm{p}=0.453$ ).

Conclusion Normalisation of serum IgG values in patients with $\mathrm{AIH}$ following treatment is not predicative of histological remission. At present, this requires a liver biopsy.

Disclosure of Interest None Declared.

\section{PTU-137 PNEUMOCOCCAL VACCINATION IN PATIENTS WITH LIVER CIRRHOSIS - IS THE MESSAGE BEING HEARD?}

V Sehgal* ${ }^{*}$ H Alexander, K Besherdas. Gastroenterology, Chase Farm Hospital, London, UK

10.1136/gutjnl-2014-307263.211 
Introduction Patients with liver cirrhosis are immunocompromised and prone to invasive infection where outcomes are usually poor. In particular, cirrhosis is a major risk factor for severe pneumococcal infection which has a mortality rate of up to $20 \%$. Whilst patients awaiting liver transplantation routinely receive the pneumococcal vaccine, it is less clear whether the remainder of patients with cirrhosis do so or not. The present literature suggests that pneumococcal vaccination prophylaxis should be administered in cirrhotic patients and revaccination desirable at 5-yearly intervals.

The aims of this study were to assess whether patients with liver cirrhosis received pneumococcal vaccination in line with current recommendations at a single centre in the UK.

Methods A retrospective analysis of all patients with biopsy-proven liver cirrhosis, irrespective of aetiology, over a 7-year period (2005-2012) at Barnet and Chase Farm Hospitals was performed. We used a database of patients with biopsy-proven cirrhosis created by our histopathology department. Patient's primary care physicians were contacted in writing to assess whether they had received the pneumococcal vaccine.

Results 37 patients had biopsy-proven liver cirrhosis over the audit period. There was no response from the primary care physicians of 6 patients. Data for 31 patients (17 male, 14 female), median age 50 years were analysed. 14 (45\%) patients had received the pneumococcal vaccine. 4 (28.5\%) of these patients were vaccinated after their histopathological diagnosis of cirrhosis was proven. The median time interval from diagnosis to vaccination was 24 months in this group of patients. Of the 7 patients vaccinated before 2007 , none had received a repeat vaccine after 5 years.

Conclusion More than half of patients with biopsy-proven liver cirrhosis in our study did not receive the pneumococcal vaccine placing these patients at risk of life threatening preventable disease. None of the patients who received the vaccine initially were revaccinated at five yearly intervals as present literature would recommend.

We would recommend an increased awareness of the importance of regular pneumococcal vaccination to all healthcare professionals, primary care physicians and those in secondary care, who come into contact with this important and growing cohort of patients.

Disclosure of Interest None Declared.

\section{PTU-138 POPULATION-BASED STUDY OF ETHNICITY AND THE DIAGNOSIS GAP IN LIVER DISEASE}

W Alazawi ${ }^{*}$, R Mathur, K Abeysekera, S Hull, K Boomla, J Robson, GR Foster. The Blizard Institute, Queen Mary, University of London, London, UK

\subsection{6/gutjnl-2014-307263.212}

Introduction Awareness of liver disease as a major cause of morbidity and mortality has led to an increase in liver function tests (LFTs) performed in primary care with abnormal results a common finding. However, we hypothesise that a large gap exists between numbers of patients with abnormal LFTs and those with recorded liver diagnoses. Non-alcoholic fatty liver disease (NAFLD) is the most common cause of chronic liver injury. Metabolic syndrome, common in people from the Indian subcontinent, is an important risk factor for NAFLD. We hypothesise that NAFLD is more common among adults of South Asian ethnicities.

Methods In a cross-sectional study of 690,683 adults, registered in co-terminus general practices in a region with high ethnic diversity, we extracted demographic information and clinical care data including LFTs in the previous two years, liver disease diagnoses and co-morbidites from the EMIS Web computerised medical records. The breakdown of age, gender, deprivation, BMI, smoking status, alcohol consumption, co-morbidities and cholesterol was described for the whole population and for the six main ethnic groups of Bangladeshi, Indian, Pakistani, White, African and Caribbean. STATA 12 was used to conduct multivariate logistic regression analyses.

Results LFTs were performed on 218,032 patients, of whom 31,627 had elevated serum transaminases. Testing varied by age, ethnicity and the presence of co-morbidities. The prevalence of abnormal LFTs was highest among Bangladeshis and independent risk factors for abnormal LFTs included male gender, alcohol consumption and elements of the metabolic syndrome.

The most commonly recorded liver diagnosis was NAFLD, followed by chronic viral infection and alcoholic liver disease. $88.4 \%$ ( $\mathrm{n}=27,985$ ) of patients with abnormal LFTs did not have a coded liver diagnosis.

The prevalence of recorded NAFLD was highest among patients of Bangladeshi ethnicity. In a multivariate analysis, independent risk factors for NAFLD included Bangladeshi ethnicity, diabetes, raised BMI, hypertension and hypercholesterolaemia.

Conclusion Abnormal LFTs are common in the population, but are under-investigated and often remain undiagnosed. Bangladeshi ethnicity is an important independent risk factor for NAFLD. Among the group of patients with abnormal LFTs and no recorded liver diagnosis, many will have a liver disease that is amenable to further management, which may prevent complications. There is a need for evidence-based guidelines for the investigation, referral and management of patients with abnormal liver tests in the community in order to ensure early identification of treatable disease.

Disclosure of Interest None Declared.

\section{PTU-139 OUTCOMES OF PATIENTS WITH LIVER CIRRHOSIS IN A NON LIVER-SPECIALIST INTENSIVE CARE UNIT: DO ADMISSION LACTATE AND APACHE 2 SCORE HELP PREDICT SUCCESSFUL DISCHARGE?}

W Waddingham*, M Cepkova. Intensive Care, Whittington Health, London, UK

\subsection{6/gutjnl-2014-307263.213}

Introduction Hospitalised patients with cirrhosis often require admission to intensive care (ITU), usually for management of secondary complications such as GI bleeding, sepsis, or organ failure. Prognosis of these patients is poor. Despite demonstration of modest improvements in recent years, overall in-hospital mortality in this group remained $54.6 \%$ in a recent study. ${ }^{1}$ Our study aimed to explore the characteristics and outcomes of cirrhotic patients admitted to ITU, and whether this was comparable to published data. We also aimed to explore whether parameters such as APACHE 2 score and admission blood lactate differ between those who survived their ITU stay and those that did not, and if this may help predict discharge.

Methods A retrospective analysis was performed of patients admitted to the Whittington hospital ITU from January 2011June 2013. Information regarding patients with a diagnosis of cirrhosis was gathered from the Intensive Care National Audit and Research unit (ICNARC) database, and discharge summaries.

Results We identified 60 patients with cirrhosis, 3.07\% of total ITU admissions, mean age 54.8 years (range 19-78). 49/60 